\title{
Global Exponential Stability of Positive Pseudo-Almost-Periodic Solutions for a Model of Hematopoiesis
}

\author{
Junxia Meng \\ College of Mathematics, Physics and Information Engineering, Jiaxing University, Jiaxing, Zhejiang 314001, China \\ Correspondence should be addressed to Junxia Meng; mengjunxia1968@aliyun.com
}

Received 11 August 2013; Accepted 16 October 2013

Academic Editor: Chuanzhi Bai

Copyright (C) 2013 Junxia Meng. This is an open access article distributed under the Creative Commons Attribution License, which permits unrestricted use, distribution, and reproduction in any medium, provided the original work is properly cited.

\begin{abstract}
This paper presents a new generalized model of hematopoiesis with multiple time-varying delays. The main purpose of this paper is to study the existence and the global exponential stability of the positive pseudo almost periodic solutions, which are more general and complicated than periodic and almost periodic solutions. Under suitable assumptions, and by using fixed point theorem, sufficient conditions are given to ensure that all solutions of this model converge exponentially to the positive pseudo almost periodic solution for the considered model. These results improve and extend some known relevant results.
\end{abstract}

\section{Introduction}

As we all know, many phenomena in nature have oscillatory character and their mathematical models have led to the introduction of certain classes of functions to describe them. For example, the pseudo almost periodic functions are the natural generalization of the concept of almost periodicity. These are functions on the real numbers set that can be represented uniquely in the form $f=h+\varphi$, where $h$ (the principal term) is an almost periodic function and $\varphi$ (the ergodic perturbation) a continuous function whose mean vanishes at infinity. Note that there exists abundant literature on the topic (see, e.g., [1-6]). In a classic study of population dynamics, the following delay differential equation model

$$
x^{\prime}(t)=-a(t) x(t)+\sum_{i=1}^{m} \frac{b_{i}(t)}{1+x^{n}\left(t-\tau_{i}(t)\right)},
$$

where $n$ is a positive constant and

$$
a, b_{i}, \tau_{i}: \mathbb{R} \longrightarrow(0,+\infty)
$$

are continuous functions for $i=1,2, \ldots, m$,

has been used by $[7,8]$ to describe the dynamics of hematopoiesis (blood cell production). As we known, (1) belongs to a class of biological systems and it (or its analogue equation) has attracted more attention to the problem of almost periodic solutions because of its extensively realistic significance. For example, some criteria ensuring the existence and stability of positive almost periodic solutions were established in [9-12] and the references cited therein. However, it is very difficult to study the global stability of positive pseudo almost periodic solution for (1). So far, no attention has been paid to the conditions for the global exponential stability on positive pseudo almost periodic solution of model (1) in terms of its coefficients. On the other hand, since the exponential convergent rate can be unveiled, the global exponential stability plays a key role in characterizing the behavior of dynamical system (see [13-15]). Thus, it is worthwhile to continue to investigate the existence and global exponential stability of positive pseudo almost periodic solutions of (1).

Motivated by the above discussions, in this paper, we consider the existence, uniqueness, and global exponential stability of positive pseudo almost periodic solutions of (1). Here in this paper, a new approach will be developed to obtain a delay-independent condition for the global exponential stability of the positive pseudo almost periodic solutions of (1), and the exponential convergent rate can be unveiled. 
Throughout this paper, for $i=1,2, \ldots, m$, it will be assumed that $a, b_{i}, \tau_{i}: \mathbb{R} \rightarrow(0,+\infty)$ are continuous functions, and

$$
\begin{gathered}
a^{-}=\inf _{t \in \mathbb{R}} a(t)>0, \quad a^{+}=\sup _{t \in \mathbb{R}} a(t), \\
b_{i}^{-}=\inf _{t \in \mathbb{R}} b_{i}(t)>0, \quad b_{i}^{+}=\sup _{t \in \mathbb{R}} b_{i}(t), \\
r=\max _{1 \leq i \leq m}\left\{\sup _{t \in \mathbb{R}} \tau_{i}(t)\right\}>0 .
\end{gathered}
$$

Let $\mathbb{R}_{+}$denote nonnegative real number space, let $C=$ $C([-r, 0], \mathbb{R})$ be the continuous functions space equipped with the usual supremum norm $\|\cdot\|$, and let $C_{+}=$ $C\left([-r, 0], \mathbb{R}_{+}\right)$. If $x(t)$ is defined on $\left[-r+t_{0}, \sigma\right)$ with $t_{0}, \sigma \in$ $\mathbb{R}$, then we define $x_{t} \in C$ where $x_{t}(\theta)=x(t+\theta)$ for all $\theta \in[-r, 0]$.

Due to the biological interpretation of model (1), only positive solutions are meaningful and therefore admissible. Thus we just consider admissible initial conditions.

$$
x_{t_{0}}=\varphi, \quad \varphi \in C_{+}, \quad \varphi(0)>0 .
$$

We write $x_{t}\left(t_{0}, \varphi\right)\left(x\left(t ; t_{0}, \varphi\right)\right)$ for an admissible solution of the admissible initial value problem (1) and (4). Also, let $\left[t_{0}, \eta(\varphi)\right)$ be the maximal right interval of existence of $x_{t}\left(t_{0}, \varphi\right)$.

\section{Preliminary Results}

In this section, some lemmas and definitions will be presented, which are of importance in proving our main results in Section 3.

In this paper, $\mathrm{BC}(\mathbb{R}, \mathbb{R})$ denotes the set of bounded continued functions from $\mathbb{R}$ to $\mathbb{R}$. Note that $\left(B C(\mathbb{R}, \mathbb{R}),\|\cdot\|_{\infty}\right)$ is a Banach space where $\|\cdot\|_{\infty}$ denotes the sup norm $\|f\|_{\infty}:=$ $\sup _{t \in \mathbb{R}}|f(t)|$.

Definition 1 (see $[16,17]$ ). Let $u(t) \in \mathrm{BC}(\mathbb{R}, \mathbb{R}) . u(t)$ is said to be almost periodic on $\mathbb{R}$ if, for any $\varepsilon>0$, the set $T(u, \varepsilon)=\{\delta$ : $|u(t+\delta)-u(t)|<\varepsilon$ for all $t \in \mathbb{R}\}$ is relatively dense; that is, for any $\varepsilon>0$, it is possible to find a real number $l=l(\varepsilon)>0$, and for any interval with length $l(\varepsilon)$, there exists a number $\delta=\delta(\varepsilon)$ in this interval such that $|u(t+\delta)-u(t)|<\varepsilon$, for all $t \in \mathbb{R}$.

We denote by $\operatorname{AP}(\mathbb{R}, \mathbb{R})$ the set of the almost periodic functions from $\mathbb{R}$ to $\mathbb{R}$. Besides, the concept of pseudo almost periodicity (pap) was introduced by Zhang in the early nineties. It is a natural generalization of the classical almost periodicity. Precisely, define the class of functions $\mathrm{PAP}_{0}(\mathbb{R}, \mathbb{R})$ as follows:

$$
\left\{f \in \mathrm{BC}(\mathbb{R}, \mathbb{R})\left|\lim _{T \rightarrow+\infty} \frac{1}{2 T} \int_{-T}^{T}\right| f(t) \mid d t=0\right\} .
$$

A function $f \in \mathrm{BC}(\mathbb{R}, \mathbb{R})$ is called pseudo almost periodic if it can be expressed as

$$
f=h+\varphi
$$

where $h \in \operatorname{AP}(\mathbb{R}, \mathbb{R})$ and $\varphi \in \operatorname{PAP}_{0}(\mathbb{R}, \mathbb{R})$. The collection of such functions will be denoted by $\operatorname{PAP}(\mathbb{R}, \mathbb{R})$. The functions $h$ and $\varphi$ in the above definition are, respectively, called the almost periodic component and the ergodic perturbation of the pseudo almost periodic function $f$. The decomposition given in definition above is unique. Observe that $\left(\operatorname{PAP}(\mathbb{R}, \mathbb{R}),\|\cdot\|_{\infty}\right)$ is a Banach space and $\operatorname{AP}(\mathbb{R}, \mathbb{R})$ is a proper subspace of $\operatorname{PAP}(\mathbb{R}, \mathbb{R})$ since the function $\phi(t)=$ $\cos \pi t+\cos t+1 /\left(1+t^{2}\right)$ is pseudo almost periodic function but not almost periodic. It should be mentioned that pseudo almost periodic functions possess many interesting properties; we will need only a few of them and for the proofs we shall refer to [16].

Lemma 2. Let $x_{1}(\cdot), \sigma(\cdot) \in A P(\mathbb{R}, \mathbb{R}), \sigma^{\prime}(\cdot) \in B C(\mathbb{R}, \mathbb{R})$ and $x_{2}(\cdot) \in P A P_{0}(\mathbb{R}, \mathbb{R})$. Then

$$
\begin{aligned}
& \text { (1) } x_{1}(t-\sigma(t)) \in A P(\mathbb{R}, \mathbb{R}) \text {; } \\
& \text { (2) } x_{2}(t-\sigma(t)) \in P A P_{0}(\mathbb{R}, \mathbb{R}) \text {, if }\left(1-\sigma^{\prime}(t)\right)^{-}=\inf _{t \in \mathbb{R}}(1- \\
& \left.\sigma^{\prime}(t)\right)>0 \text {. }
\end{aligned}
$$

Proof. (1) For any $\varepsilon>0$, from the uniform continuity of $x_{1}(\cdot)$, we can choose a constant

$$
0<\delta=\delta(\varepsilon)<\frac{\varepsilon}{2}
$$

such that

$$
\left|x_{1}\left(t^{\prime}\right)-x_{1}\left(t^{\prime \prime}\right)\right|<\frac{\varepsilon}{2}, \quad \forall t^{\prime}, t^{\prime \prime} \in \mathbb{R},\left|t^{\prime}-t^{\prime \prime}\right|<\delta .
$$

From the theory of almost periodic functions in $[16,17]$, it follows that for $\delta>0$, it is possible to find a real number $l=l(\delta)=l(\delta(\varepsilon))>0$, and for any interval with length $l$, there exists a number $\tau=\tau(\varepsilon)$ in this interval such that

$$
\begin{array}{r}
|\sigma(t+\tau)-\sigma(t)|<\delta, \quad\left|x_{1}(t+\tau)-x_{1}(t)\right|<\delta<\frac{\varepsilon}{2} \\
\forall t \in \mathbb{R} .
\end{array}
$$

Combing (8) and (9), we obtain

$$
\begin{aligned}
& \left|x_{1}(t+\tau-\sigma(t+\tau))-x_{1}(t-\sigma(t))\right| \\
& \leq\left|x_{1}(t+\tau-\sigma(t+\tau))-x_{1}(t+\tau-\sigma(t))\right| \\
& \quad+\left|x_{1}(t+\tau-\sigma(t))-x_{1}(t-\sigma(t))\right| \\
& <\frac{\varepsilon}{2}+\frac{\varepsilon}{2}=\varepsilon, \quad \forall t \in \mathbb{R} .
\end{aligned}
$$

which yields $x_{1}(t-\sigma(t)) \in \mathrm{AP}(\mathbb{R}, \mathbb{R})$. 
(2) Set $s=t-\sigma(t)$; we get

$$
\begin{aligned}
0 & \leq \frac{1}{2 T} \int_{-T}^{T}\left|x_{2}(t-\sigma(t))\right| d t \\
& =\frac{1}{2 T} \int_{-(T-\sigma(-T))}^{T-\sigma(T)}\left|x_{2}(s)\right| \frac{1}{1-\sigma^{\prime}(t)} d s \\
& \leq \frac{1}{\left(1-\sigma^{\prime}(t)\right)^{-}} \frac{T+\sigma^{+}}{T} \frac{1}{2\left(T+\sigma^{+}\right)} \int_{-\left(T+\sigma^{+}\right)}^{T+\sigma^{+}}\left|x_{2}(s)\right| d s, \\
\text { where } \sigma^{+} & =\sup _{t \in \mathbb{R}} \sigma(t),
\end{aligned}
$$

which implies that $x_{2}(t-\sigma(t)) \in \mathrm{PAP}_{0}(\mathbb{R}, \mathbb{R})$.

Remark 3. Set $x(\cdot)=x_{1}(\cdot)+x_{2}(\cdot)$ with $x_{1}(\cdot) \in \mathrm{AP}(\mathbb{R}, \mathbb{R})$ and $x_{2}(\cdot) \in \mathrm{PAP}_{0}(\mathbb{R}, \mathbb{R})$. It follows from Lemma 2 that

$$
\begin{gathered}
x(t-\sigma(t)) \in \operatorname{PAP}(\mathbb{R}, \mathbb{R}), \quad \text { if }\left(1-\sigma^{\prime}(t)\right)^{-}>0, \\
\sigma(\cdot) \in \mathrm{AP}(\mathbb{R}, \mathbb{R}), \quad \sigma^{\prime}(\cdot) \in \mathrm{BC}(\mathbb{R}, \mathbb{R}) .
\end{gathered}
$$

Definition 4 (see $[16,17])$. Let $x \in R^{n}$ and let $Q(t)$ be an $n \times n$ continuous matrix defined on $R$. The linear system

$$
x^{\prime}(t)=Q(t) x(t)
$$

is said to admit an exponential dichotomy on $R$ if there exist positive constants $k$, and $\alpha$, projection $P$, and the fundamental solution matrix $X(t)$ of (13) satisfying

$$
\begin{aligned}
& \left\|X(t) P X^{-1}(s)\right\| \leq k e^{-\alpha(t-s)} \quad \text { for } t \geq s, \\
& \left\|X(t)(I-P) X^{-1}(s)\right\| \leq k e^{-\alpha(s-t)} \quad \text { for } t \leq s .
\end{aligned}
$$

Lemma 5 (see $[6,16]$ ). Assume that $Q(t)$ is an almost periodic matrix function and $g(t) \in P A P\left(R^{n}\right)$. If the linear system (13) admits an exponential dichotomy, then pseudo almost periodic system

$$
x^{\prime}(t)=Q(t) x+g(t)
$$

has a unique pseudo almost periodic solution $x(t)$, and

$$
\begin{aligned}
x(t)= & \int_{-\infty}^{t} X(t) P X^{-1}(s) g(s) d s \\
& -\int_{t}^{+\infty} X(t)(I-P) X^{-1}(s) g(s) d s .
\end{aligned}
$$

Lemma 6 (see $[16,17])$. Let $c_{i}(t)$ be an almost periodic function on $R$ and

$$
M\left[c_{i}\right]=\lim _{T \rightarrow+\infty} \frac{1}{T} \int_{t}^{t+T} c_{i}(s) d s>0, \quad i=1,2, \ldots, n .
$$

Then the linear system

$$
x^{\prime}(t)=\operatorname{diag}\left(-c_{1}(t),-c_{2}(t), \ldots,-c_{n}(t)\right) x(t)
$$

admits an exponential dichotomy on $R$.
Lemma 7 (see [11, Lemma 2.3]). Every solution $x\left(t ; t_{0}, \varphi\right)$ of (1) and (4) is positive and bounded on $\left[t_{0}, \eta(\varphi)\right)$, and $\eta(\varphi)=$ $+\infty$.

Lemma 8. Suppose that there exist two positive constants $\kappa$ and $M$ such that

$$
\begin{gathered}
M>\kappa, \quad \sup _{t \in R}\left\{-a(t) M+\sum_{i=1}^{m} b_{i}(t)\right\}<0, \\
\inf _{t \in R}\left\{-a(t) \kappa+\sum_{i=1}^{m} \frac{b_{i}(t)}{1+M^{n}}\right\}>0 .
\end{gathered}
$$

Then, there exists $t_{\varphi}>t_{0}$ such that

$$
\kappa<x\left(t ; t_{0}, \varphi\right)<M, \quad \forall t \geq t_{\varphi}
$$

Proof. This Lemma can be proven in a similar way to that in Lemma 2.2 of [12]. But for convenience of reading, we give the proof as follows. Let $x(t)=x\left(t ; t_{0}, \varphi\right)$. We first claim that there exists $t^{\#} \in\left[t_{0},+\infty\right)$ such that

$$
x\left(t^{\#}\right)<M
$$

Otherwise,

$$
x(t) \geq M, \quad \forall t \in\left[t_{0},+\infty\right)
$$

Which, together with (19), implies that

$$
\begin{aligned}
x^{\prime}(t) & =-a(t) x(t)+\sum_{i=1}^{m} \frac{b_{i}(t)}{1+x^{n}\left(t-\tau_{i}(t)\right)} \\
& \leq-a(t) M+\sum_{i=1}^{m} \frac{b_{i}(t)}{1+M^{n}} \\
& \leq-a(t) M+\sum_{i=1}^{m} b_{i}(t) \\
& \leq \sup _{t \in R}\left\{-a(t) M+\sum_{i=1}^{m} b_{i}(t)\right\} \\
& <0, \quad \forall t \geq t_{0}+r .
\end{aligned}
$$

This yields that

$$
\begin{aligned}
x(t)= & x\left(t_{0}+r\right)+\int_{t_{0}+r}^{t} x^{\prime}(s) d s \\
\leq & x\left(t_{0}+r\right)+\sup _{t \in R}\left\{-a(t) M+\sum_{i=1}^{m} b_{i}(t)\right\} \\
& \times\left(t-\left(t_{0}+r\right)\right), \quad \forall t \geq t_{0}+r .
\end{aligned}
$$

Thus

$$
\lim _{t \rightarrow+\infty} x(t)=-\infty
$$


which contradicts the fact that $x(t)$ is positive and bounded on $\left[t_{0},+\infty\right)$. Hence, (21) holds. In the sequel, we prove that

$$
x(t)<M, \quad \forall t \in\left[t^{\#},+\infty\right) .
$$

Suppose, for the sake of contradiction, there exists $\tilde{t} \in\left(t^{\#}\right.$, $+\infty)$ such that

$$
x(\tilde{t})=M, \quad x(t)<M, \quad \forall t \in\left[t^{\#}, \tilde{t}\right) .
$$

Calculating the derivative of $x(t)$, together with (19), (1), and (27), implies that

$$
\begin{aligned}
0 & \leq x^{\prime}(\widetilde{t})=-a(\widetilde{t}) x(\widetilde{t})+\sum_{i=1}^{m} \frac{b_{i}(\widetilde{t})}{1+x^{n}\left(\widetilde{t}-\tau_{i}(\widetilde{t})\right)} \\
& \leq-a(\widetilde{t}) M+\sum_{i=1}^{m} b_{i}(\widetilde{t})<0,
\end{aligned}
$$

which is a contradiction and implies that (26) holds.

We finally show that $l=\liminf _{t \rightarrow \infty} x(t)>\kappa$. By way of contradiction, we assume that $0 \leq l \leq \kappa$. By the fluctuation lemma [18, Lemma A.1.], there exists a sequence $\left\{t_{k}\right\}_{k \geq 1}$ such that

$$
\begin{aligned}
& t_{k} \longrightarrow+\infty, \quad x\left(t_{k}\right) \longrightarrow \liminf _{t \rightarrow+\infty} x(t), \\
& x^{\prime}\left(t_{k}\right) \longrightarrow 0 \quad \text { as } k \longrightarrow+\infty .
\end{aligned}
$$

Since $\left\{x_{t_{k}}\right\}$ is bounded and equicontinuous, by the AscoliArzelá theorem, there exists a subsequence, still denoted by itself for simplicity of notation, such that

$$
x_{t_{k}} \longrightarrow \varphi^{*}(k \longrightarrow+\infty) \text { for some } \varphi^{*} \in C_{+} .
$$

Moreover,

$$
\varphi^{*}(0)=l \leq \varphi^{*}(\theta) \leq M \quad \text { for } \theta \in[-r, 0) .
$$

Without loss of generality, we assume that all $a\left(t_{k}\right), b_{i}\left(t_{k}\right)$, and $\tau_{i}\left(t_{k}\right)$ are convergent to $a^{*}, b_{i}^{*}$, and $\tau_{i}^{*}$, respectively. This can be achieved because of almost periodicity. It follows from

$$
x^{\prime}\left(t_{k}\right)=-a\left(t_{k}\right) x\left(t_{k}\right)+\sum_{i=1}^{m} \frac{b_{i}\left(t_{k}\right)}{1+x^{n}\left(t_{k}-\tau_{i}\left(t_{k}\right)\right)}
$$

that (taking limits)

$$
\begin{aligned}
0 & =-a^{*} l+\sum_{i=1}^{m} \frac{b_{i}^{*}}{1+\left(\varphi^{*}\left(-\tau_{i}^{*}\right)\right)^{n}} \\
& \geq-a^{*} l+\sum_{i=1}^{m} \frac{b_{i}^{*}}{1+M^{n}} \\
& \geq-a^{*} \kappa+\sum_{i=1}^{m} \frac{b_{i}^{*}}{1+M^{n}} \\
& \geq \inf _{t \in R}\left\{-a(t) \kappa+\sum_{i=1}^{m} \frac{b_{i}(t)}{1+M^{n}}\right\}>0,
\end{aligned}
$$

is a contradiction. This proves that $l>\kappa$. Hence, from (26), we can choose $t_{\varphi}>t_{0}$ such that

$$
\kappa<x\left(t ; t_{0}, \varphi\right)<M, \quad \forall t \geq t_{\varphi} .
$$

This ends the proof of Lemma 8.

\section{Main Results}

Theorem 9. Suppose that

$a, \tau_{i} \in A P(\mathbb{R}, \mathbb{R}), \quad \tau_{i}^{\prime}(\cdot) \in B C(\mathbb{R}, \mathbb{R}), \quad b_{i} \in P A P(\mathbb{R}, \mathbb{R})$,

$$
\inf _{t \in \mathbb{R}}\left(1-\tau_{i}^{\prime}(t)\right)>0, \quad i=1,2, \ldots, m,
$$

and there exist two positive constants $\kappa$ and $M$ satisfying (19) and

$$
\sup _{t \in R}\left\{-a(t)+\sum_{i=1}^{m} b_{i}(t) \frac{n}{4 \kappa}\right\}<0
$$

Then, there exists a unique positive pseudo almost periodic solution of (1) in the region $B^{*}=\{\varphi \mid \varphi \in P A P(\mathbb{R}, \mathbb{R}), \kappa \leq$ $\varphi(t) \leq M$, for all $t \in \mathbb{R}\}$.

Proof. Consider $\Upsilon:[0 ; 1] \rightarrow \mathbb{R}$ defined by

$$
\Upsilon(u)=\sup _{t \in R}\left\{-a(t)+\sum_{i=1}^{m} b_{i}(t) \frac{n}{4 \kappa} e^{u}\right\}, \quad u \in[0,1] .
$$

Then, we have

$$
\Upsilon(0)=\sup _{t \in R}\left\{-a(t)+\sum_{i=1}^{m} b_{i}(t) \frac{n}{4 \kappa}\right\}<0,
$$

which implies that there exists a constant $\varsigma \in(0,1]$ such that

$$
\Upsilon(\varsigma)=\sup _{t \in R}\left\{-a(t)+\sum_{i=1}^{m} b_{i}(t) \frac{n}{4 \kappa} e^{\varsigma}\right\}<0 .
$$

For any $\phi \in \operatorname{PAP}(\mathbb{R}, \mathbb{R})$, from (35), Remark 3, and the composition theorem of pseudo almost periodic functions [16], we have

$$
\sum_{i=1}^{m} \frac{b_{i}(t)}{1+\phi^{n}\left(t-\tau_{i}(t)\right)} \in \operatorname{PAP}(\mathbb{R}, \mathbb{R})
$$

We next consider an auxiliary equation:

$$
x^{\prime}(t)=-a(t) x(t)+\sum_{i=1}^{m} \frac{b_{i}(t)}{1+\phi^{n}\left(t-\tau_{i}(t)\right)} .
$$

Notice that $M[a]>0$; it follows from Lemma 6 that the linear equation

$$
x^{\prime}(t)=-a(t) x(t)
$$

admits an exponential dichotomy on $\mathbb{R}$. Thus, by Lemma 5 , we obtain that the system (41) has exactly one pseudo almost periodic solution:

$$
x^{\phi}(t)=\int_{-\infty}^{t} e^{-\int_{s}^{t} a(u) d u}\left[\sum_{i=1}^{m} \frac{b_{i}(s)}{1+\phi^{n}\left(t-\tau_{i}(s)\right)}\right] d s .
$$


Define a mapping $T: \operatorname{PAP}(\mathbb{R}, \mathbb{R}) \rightarrow \operatorname{PAP}(\mathbb{R}, \mathbb{R})$ by setting

$$
T(\phi(t))=x^{\phi}(t), \quad \forall \phi \in \operatorname{PAP}(\mathbb{R}, \mathbb{R}) .
$$

Since $B^{*}=\{\varphi \mid \varphi \in \operatorname{PAP}(\mathbb{R}, \mathbb{R}), \kappa \leq \varphi(t) \leq M$, for all $t \in \mathbb{R}\}$, it is easy to see that $B^{*}$ is a closed subset of $\operatorname{PAP}(R, R)$. For any $\phi \in B^{*}$, from (19), we have

$$
\begin{aligned}
x^{\phi}(t) & \leq \int_{-\infty}^{t} e^{-\int_{s}^{t} a(u) d u}\left[\sum_{i=1}^{m} b_{i}(s)\right] d s \\
& \leq \int_{-\infty}^{t} e^{-\int_{s}^{t} a(u) d u} a(s) M d s=M, \quad \forall t \in R, \\
x^{\phi}(t) & \geq \int_{-\infty}^{t} e^{-\int_{s}^{t} a(u) d u}\left[\sum_{i=1}^{m} \frac{b_{i}(s)}{1+M^{n}}\right] d s \\
& \geq \int_{-\infty}^{t} e^{-\int_{s}^{t} a(u) d u} a(s) \kappa d s=\kappa, \quad \forall t \in R .
\end{aligned}
$$

This implies that the mapping $T$ is a self-mapping from $B^{*}$ to $B^{*}$. Now, we prove that the mapping $T$ is a contraction mapping on $B^{*}$. In fact, for $\varphi, \psi \in B^{*}$, we get

$$
\begin{aligned}
&\|T(\varphi)-T(\psi)\|_{\infty} \\
&=\sup _{t \in R}|T(\varphi)(t)-T(\psi)(t)| \\
&=\sup _{t \in R} \mid \int_{-\infty}^{t} e^{-\int_{s}^{t} a(u) d u \sum_{i=1}^{m} b_{i}(s)}\left[\frac{1}{1+\varphi^{n}\left(t-\tau_{i}(s)\right)}\right. \\
&\left.\quad-\frac{1}{1+\psi^{n}\left(s-\tau_{i}(s)\right)}\right] d s \mid .
\end{aligned}
$$

In view of (39), (45), (46), and (47), from the inequality

$$
\begin{aligned}
\left|\frac{1}{1+x^{n}}-\frac{1}{1+y^{n}}\right| & =\left|\frac{-n \theta^{n-1}}{\left(1+\theta^{n}\right)^{2}}\right||x-y| \\
& \leq \frac{n \theta^{n-1}}{\left(2 \sqrt{\theta^{n}}\right)^{2}}|x-y| \leq \frac{n}{4 \kappa}|x-y|,
\end{aligned}
$$

where $x, y \in[\kappa, M]$ and $\theta$ lies between $x$ and $y$, we have

$$
\begin{aligned}
\|T(\varphi)-T(\psi)\|_{\infty} \\
\leq \sup _{t \in R} \int_{-\infty}^{t} e^{-\int_{s}^{t} a(u) d u} \sum_{i=1}^{m} b_{i}(s) \frac{n}{4 \kappa} \\
\quad \times\left|\varphi\left(s-\tau_{i}(s)\right)-\psi\left(s-\tau_{i}(s)\right)\right| d s \\
\leq \sup _{t \in R} \int_{-\infty}^{t} e^{-\int_{s}^{t} a(u) d u} a(s) e^{-\varsigma} \\
\quad \times\left|\varphi\left(s-\tau_{i}(s)\right)-\psi\left(s-\tau_{i}(s)\right)\right| d s \\
\leq e^{-\varsigma}\|\varphi-\psi\|_{\infty} .
\end{aligned}
$$

Noting that $e^{-\varsigma}<1$, it is clear that the mapping $T$ is a contraction on $B^{*}$. Using Theorem 0.3 .1 of [19], we obtain that the mapping $T$ possesses a unique fixed point $\varphi^{*} \in B^{*}$, $T \varphi^{*}=\varphi^{*}$. By (41), $\varphi^{*}$ satisfies (1). So $\varphi^{*}$ is a positive pseudo almost periodic solution of (1) in $B^{*}$. The proof of Theorem 9 is now complete.

Theorem 10. Under the assumptions of Theorem 9, (1) has at least one positive pseudo almost periodic solution $x^{*}(t)$. Moreover, $x^{*}(t)$ is globally exponentially stable; that is, there exist constants $K_{\varphi, x^{*}}, t_{\varphi, x^{*}}$, and $\lambda>0$ such that

$$
\left|x\left(t ; t_{0}, \varphi\right)-x^{*}(t)\right|<K_{\varphi, x^{*}} e^{-\lambda t}, \quad \forall t>t_{\varphi, x^{*}} .
$$

Proof. By Theorem 9, (1) has a positive pseudo almost periodic solution; say $x^{*}(t)$. It suffices to show that $x^{*}(t)$ is globally exponentially stable. Define a continuous function $\Gamma(u)$ by setting

$$
\Gamma(u)=\sup _{t \in R}\left\{-[a(t)-u]+\sum_{i=1}^{m} b_{i}(t) \frac{n}{4 \kappa} e^{r u}\right\}, \quad u \in[0,1] .
$$

Then, we have

$$
\Gamma(0)=\sup _{t \in R}\left\{-a(t)+\sum_{i=1}^{m} b_{i}(t) \frac{n}{4 \kappa}\right\}<0,
$$

which implies that there exist two constants $\eta>0$ and $\lambda \epsilon$ $(0,1]$ such that

$$
\Gamma(\lambda)=\sup _{t \in R}\left\{-[a(t)-\lambda]+\sum_{i=1}^{m} b_{i}(t) \frac{n}{4 \kappa} e^{\lambda r}\right\}<-\eta<0 .
$$

Let $x(t)=x\left(t ; t_{0}, \varphi\right)$ and $y(t)=x(t)-x^{*}(t)$, where $t \epsilon$ $\left[t_{0}-r,+\infty\right)$. Then

$$
\begin{aligned}
y^{\prime}(t)=-a(t) y(t)+\sum_{i=1}^{m} b_{i}(t)[ & \frac{1}{1+x^{n}\left(t-\tau_{i}(t)\right)} \\
& \left.-\frac{1}{1+x^{* n}\left(t-\tau_{i}(t)\right)}\right] .
\end{aligned}
$$
that

It follows from Lemma 8 that there exists $t_{\varphi, x^{*}}>t_{0}$ such

$$
\kappa \leq x(t), \quad x^{*}(t) \leq M, \quad \forall t \in\left[t_{\varphi, x^{*}}-r,+\infty\right) .
$$

We consider the Lyapunov functional

$$
V(t)=|y(t)| e^{\lambda t}
$$


Calculating the upper left derivative of $V(t)$ along the solution $y(t)$ of (54), we have

$$
\begin{aligned}
D^{-}( & V(t)) \\
\leq & -a(t)|y(t)| e^{\lambda t}+\sum_{i=1}^{m} b_{i}(t) \\
& \times\left|\frac{1}{1+x^{n}\left(t-\tau_{i}(t)\right)}-\frac{1}{1+x^{* n}\left(t-\tau_{i}(t)\right)}\right| e^{\lambda t} \\
& +\lambda|y(t)| e^{\lambda t} \\
= & {\left[-(a(t)-\lambda)|y(t)|+\sum_{i=1}^{m} b_{i}(t)\right.} \\
& \left.\times\left|\frac{1}{1+x^{n}\left(t-\tau_{i}(t)\right)}-\frac{1}{1+x^{* n}\left(t-\tau_{i}(t)\right)}\right|\right] e^{\lambda t},
\end{aligned}
$$

We claim that

$$
\begin{aligned}
V(t) & =|y(t)| e^{\lambda t} \\
& <e^{\lambda t_{\varphi, x^{*}}}\left(\max _{t \in\left[t_{0}-r, t_{\varphi, x^{*}}\right]}\left|x(t)-x^{*}(t)\right|+1\right) \\
& :=K_{\varphi, x^{*}}, \quad \forall t>t_{\varphi, x^{*}} .
\end{aligned}
$$

Contrarily, there must exists $t_{*}>t_{\varphi, x^{*}}$ such that

$$
V\left(t_{*}\right)=K_{\varphi, x^{*}}, \quad V(t)<K_{\varphi, x^{*}}, \quad \forall t \in\left[t_{0}-r, t_{*}\right) .
$$

Together with (48), (57), and (59), we obtain

$$
\begin{aligned}
0 \leq & D^{-}\left(V\left(t_{*}\right)\right) \\
\leq & {\left[-\left(a\left(t_{*}\right)-\lambda\right)\left|y\left(t_{*}\right)\right|+\sum_{i=1}^{m} b_{i}\left(t_{*}\right)\right.} \\
& \times \mid \frac{1}{1+x^{n}\left(t_{*}-\tau_{i}\left(t_{*}\right)\right)} \\
\leq & -\left(a\left(t_{*}\right)-\lambda\right)\left|y\left(t_{*}\right)\right| e^{\lambda t_{*}}+\sum_{i=1}^{m} b_{i}\left(t_{*}\right) \\
& \times \frac{n}{4 \kappa} e^{\lambda \tau_{i}\left(t_{*}\right)} e^{\lambda\left(t_{*}-\tau_{i}\left(t_{*}\right)\right)}\left|y\left(t_{*}-\tau_{i}\left(t_{*}\right)\right)\right| \\
\leq & \left\{\begin{array}{l}
\left.-\left(a\left(t_{*}\right)-\lambda\right)+\sum_{i=1}^{m} b_{i}\left(t_{*}\right) \frac{n}{4 \kappa} e^{\lambda r}\right\} K_{\varphi, x^{*}} \\
\end{array}\right.
\end{aligned}
$$

Thus,

$$
0 \leq-\left(a\left(t_{*}\right)-\lambda\right)+\sum_{i=1}^{m} b_{i}\left(t_{*}\right) \frac{n}{4 \kappa} e^{\lambda r}
$$

which contradicts (53). Hence, (58) holds. It follows that

$$
|y(t)|<K_{\varphi, x^{*}} e^{-\lambda t} \quad \forall t>t_{\varphi, x^{*}} .
$$

This completes the proof of Theorem 10 .

\section{An Example}

In this section, we present an example to check the validity of the results we obtained in the previous sections.

Example 1. Consider the following model of hematopoiesis with multiple time-varying delays:

$$
\begin{aligned}
x^{\prime}(t)= & -1.3 x(t)+\frac{1}{2}\left(2+\frac{1}{2}|\cos \sqrt{2} t|+\frac{1}{100} \frac{1}{1+t^{2}}\right) \\
& \times \frac{1}{1+x\left(t-2 e^{(1 / 10) \cos t}\right)}+\frac{1}{2} \\
& \times\left(2+\frac{1}{2}|\sin t|+\frac{1}{100} \frac{1}{1+t^{2}}\right) \frac{1}{1+x\left(t-2 e^{(1 / 10) \sin t}\right)} .
\end{aligned}
$$

Obviously

$$
\begin{gathered}
a^{+}=a^{-}=1.3, \quad b_{1}^{-}=b_{2}^{-}=1, \quad b_{1}^{+}=b_{2}^{+}=1.26, \\
n=1, \quad r=2 e^{1 / 10} .
\end{gathered}
$$

Let $\kappa=0.5$ and $M=2$. Then

$$
\begin{aligned}
-a^{-} M+b_{1}^{+}+b_{2}^{+} & =-0.08<0, \\
-a^{+} \kappa+\frac{b_{1}^{-}+b_{2}^{-}}{1+M} & =\frac{1}{60}>0, \\
-a^{-}+\left(b_{1}^{+}+b_{2}^{+}\right) \frac{n}{4 \kappa} & =-1.3+2.52 \times \frac{1}{2} \\
& =-0.04<0,
\end{aligned}
$$

which imply that (63) satisfies the assumptions of Theorem 10. Therefore, (63) has a unique positive pseudo almost periodic solution $x^{*}(t)$, which is globally exponentially stable with the exponential convergent rate $\lambda \approx 0.01$. The numerical simulation in Figure 1 strongly supports the conclusion.

Remark 11. We remark that the results in [9-12] give no opinions about global exponential convergence for the positive pseudo almost periodic solution. Thus, the results in [912] and the references therein cannot be applied to prove the global exponential stability of positive pseudo almost periodic solution for (63). This implies that the results of this paper are new and they complement previously known results.

\section{Conflict of Interests}

The author declares no conflict of interests. She also declares that she has no financial or personal relationships with other 


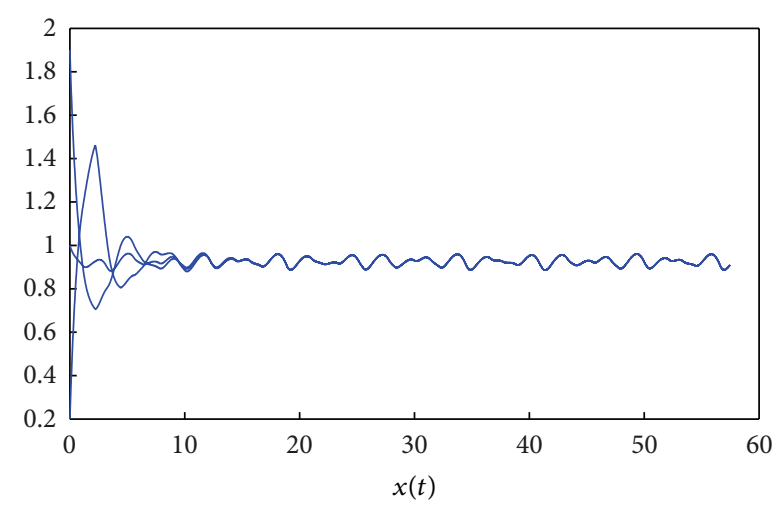

FIGURE 1: Numerical solution $x(t)$ of (63) for initial value $\varphi(t) \equiv$ $0.2,1,1.9$.

people or organizations that can inappropriately influence her work. There are no professional or other personal interests of any nature or kind in any product, service, and/or company that could be construed as influencing the position presented in, or the review of, this paper.

\section{Acknowledgments}

The author would like to express the sincere appreciation to the editor and reviewer for their helpful comments in improving the presentation and quality of the paper. In particular, the author expresses the sincere gratitude to Professor Zhibin Chen and Professor Bingwen Liu for the helpful discussion when this revision work was being carried out. This work was supported by the National Natural Science Foundation of China (Grant no. 11201184), the Natural Scientific Research Fund of Zhejiang Provincial of P.R. China (Grant nos. Y6110436 and LY12A01018), and the Natural Scientific Research Fund of Zhejiang Provincial Education Department of P.R. China (Grant no. Z201122436).

\section{References}

[1] T. Diagana and E. M. Hernández, "Existence and uniqueness of pseudo almost periodic solutions to some abstract partial neutral functional-differential equations and applications," Journal of Mathematical Analysis and Applications, vol. 327, no. 2, pp. 776-791, 2007.

[2] H. Li, F. Huang, and J. Li, "Composition of pseudo almost-periodic functions and semilinear differential equations," Journal of Mathematical Analysis and Applications, vol. 255, no. 2, pp. 436446, 2001.

[3] E. A. Dads, P. Cieutat, and L. Lhachimi, "Positive pseudo almost periodic solutions for some nonlinear infinite delay integral equations," Mathematical and Computer Modelling, vol. 49, no. 3-4, pp. 721-739, 2009.

[4] C. Zhang, "Vector-valued pseudo almost periodic functions," Czechoslovak Mathematical Journal, vol. 47, no. 3, pp. 385-394, 1997.

[5] C. Zhang, "Pseudo-almost-periodic solutions of some differential equations I," Journal of Mathematical Analysis and Applications, vol. 181, no. 1, pp. 62-76, 1994.
[6] C. Zhang, "Pseudo almost periodic solutions of some differential equations II," Journal of Mathematical Analysis and Applications, vol. 192, no. 2, pp. 543-561, 1995.

[7] M. C. Mackey and L. Glass, "Oscillations and chaos in physiological control systems," Sciences, vol. 197, pp. 287-289, 1977.

[8] I. Gyori and G. Ladas, Oscillation Theory of Delay Differential Equations with Applications, Clarendon, Oxford, UK, 1991.

[9] X. Yang, "Existence and global attractivity of unique positive almost periodic solution for a model of hematopoiesis," Applied Mathematics, vol. 25, no. 1, pp. 25-34, 2010.

[10] J. O. Alzabut, J. J. Nieto, and G. Tr. Stamov, "Existence and exponential stability of positive almost periodic solutions for a model of hematopoiesis," Boundary Value Problems, vol. 2009, Article ID 127510, 2009.

[11] H. Zhang, L. Wang, and M. Yang, "Existence and exponential convergence of the positive almost periodic solution for a model of hematopoiesis," Applied Mathematics Letters, vol. 26, no. 1, pp. 38-42, 2013.

[12] Z. Chen, "Global exponential stability of positive almost periodic solutions for a model of hematopoiesis," Kodai Mathematical Journal, vol. 37, 2014.

[13] B. Liu, "Positive periodic solutions for a nonlinear density dependent mortality Nicholson's blowflies model," Kodai Mathematical Journal, vol. 37, no. 1, 2014.

[14] B. Liu, "Global exponential stability of positive periodic solutions for a delayed Nicholson's blowflies model," Journal of Mathematical Analysis and Applications, 2013.

[15] H. Zhang and J. Shao, "Existence and exponential stability of almost periodic solutions for $\mathrm{CNNs}$ with time-varying leakage delays," Neurocomputing, vol. 121, pp. 226-233, 2013.

[16] C. Zhang, Almost Periodic Type Functions and Ergodicity, Kluwer Academic, Science Press, Beijing, China, 2003.

[17] C. Y. He, Almost Periodic Differential Equation, Higher Education Publishing House, Beijing, China, 1992 Chinese.

[18] H. L. Smith, An Introduction to Delay Differential Equations with Applications to the Life Sciences, vol. 57, Springer, New York, NY, USA, 2011.

[19] J. K. Hale, Ordinary Differential Equations, Krieger, Malabar, Fla, USA, 1980. 


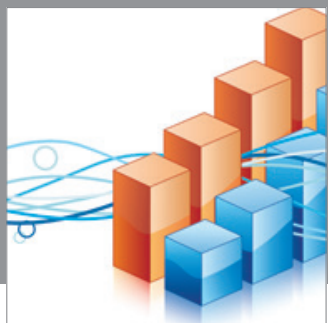

Advances in

Operations Research

mansans

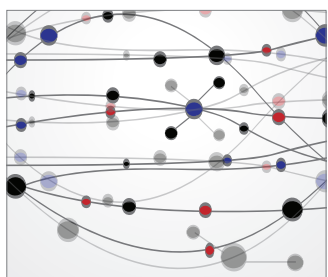

The Scientific World Journal
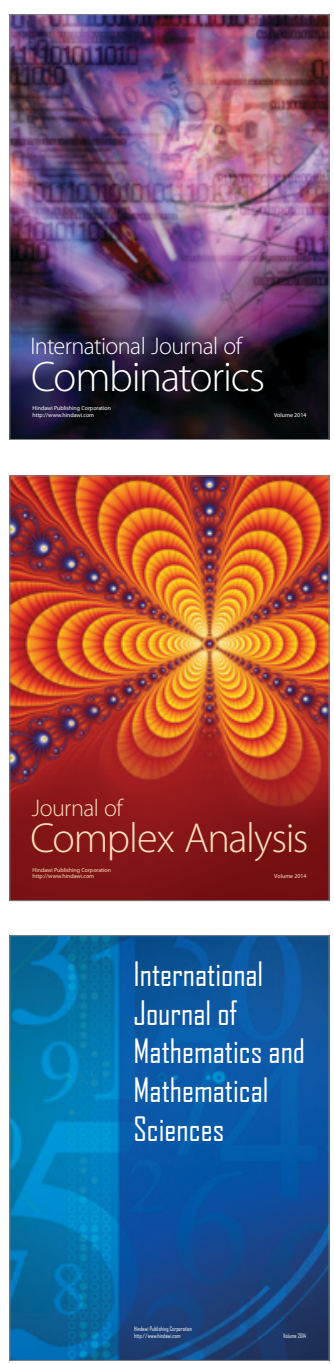
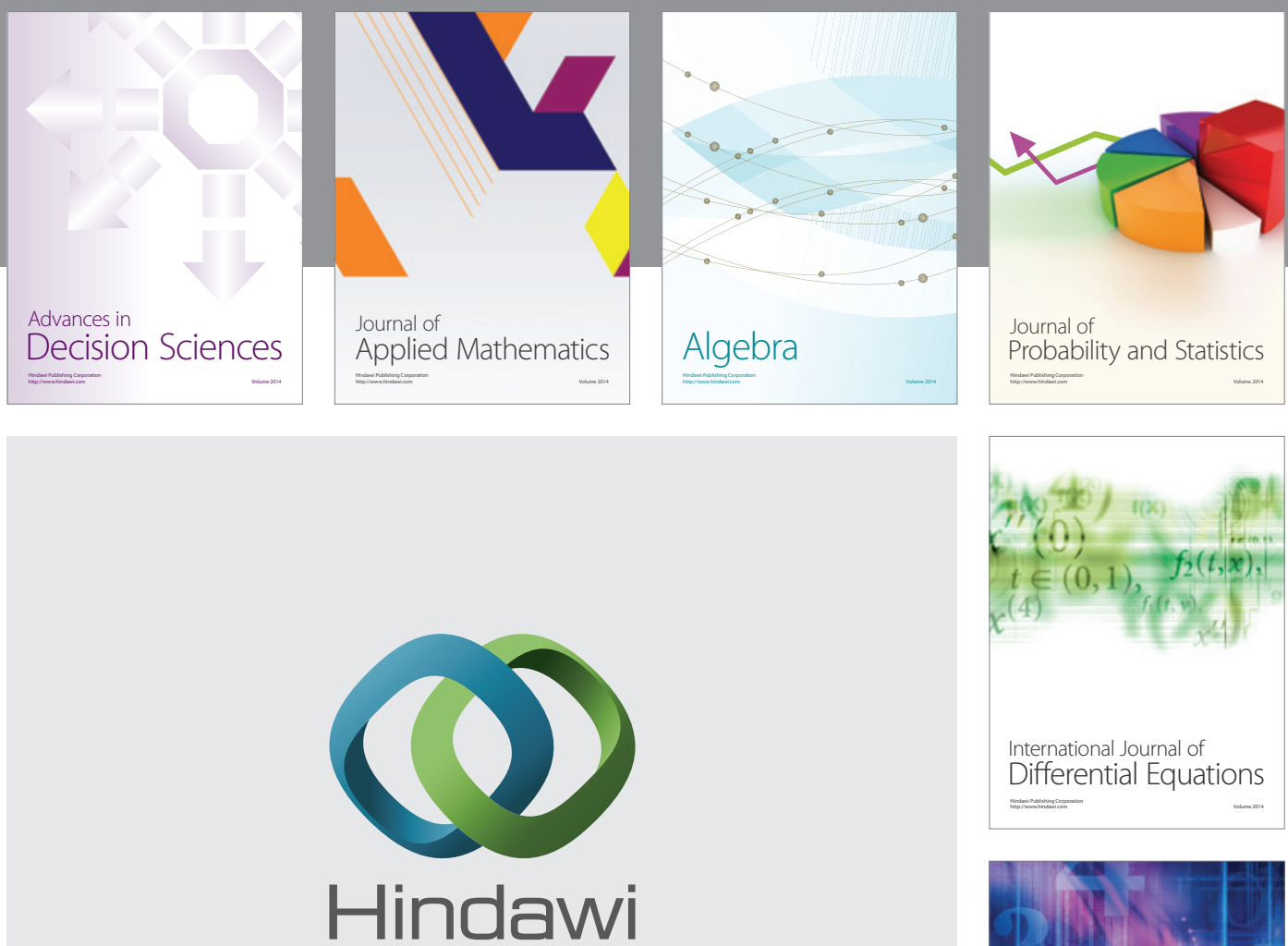

Submit your manuscripts at http://www.hindawi.com
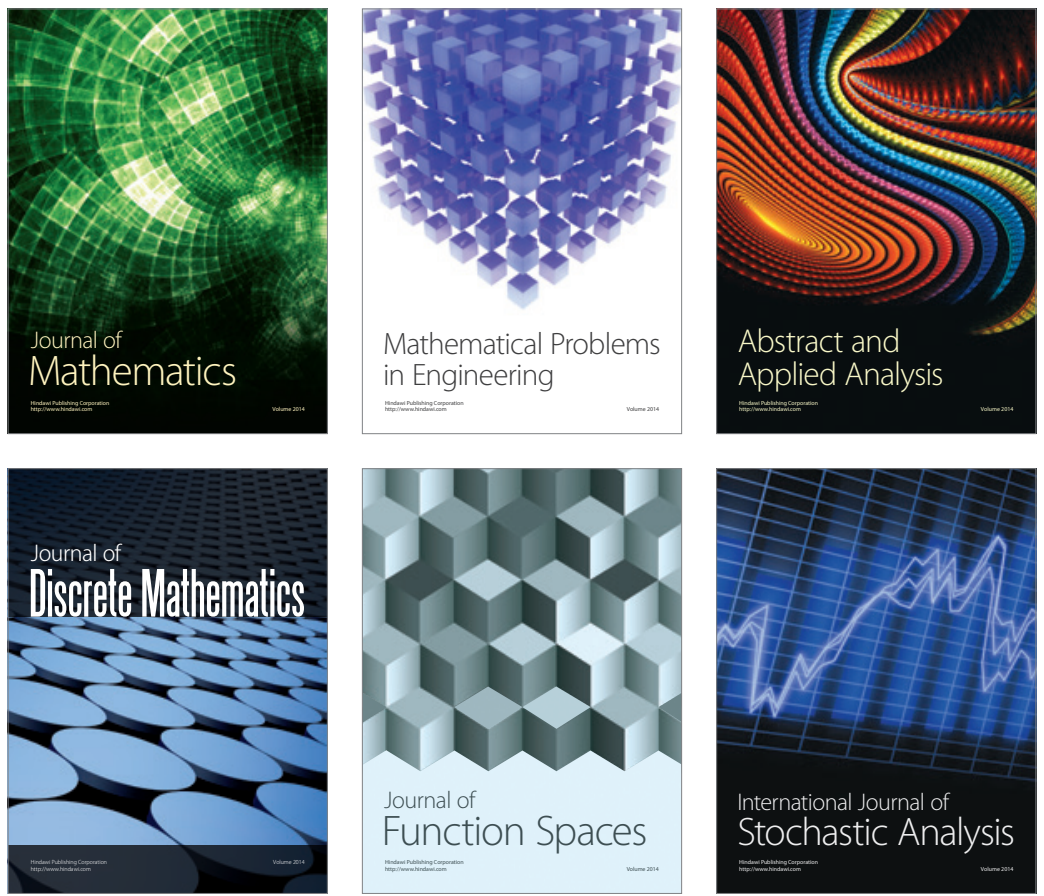

Journal of

Function Spaces

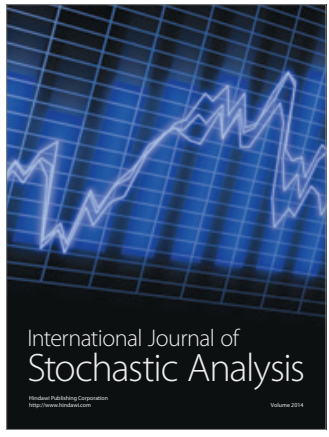

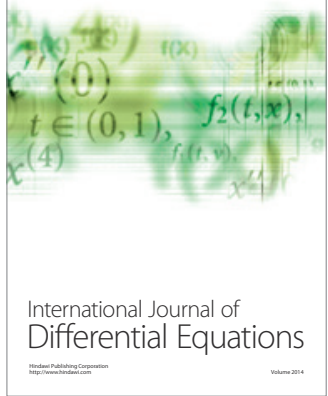
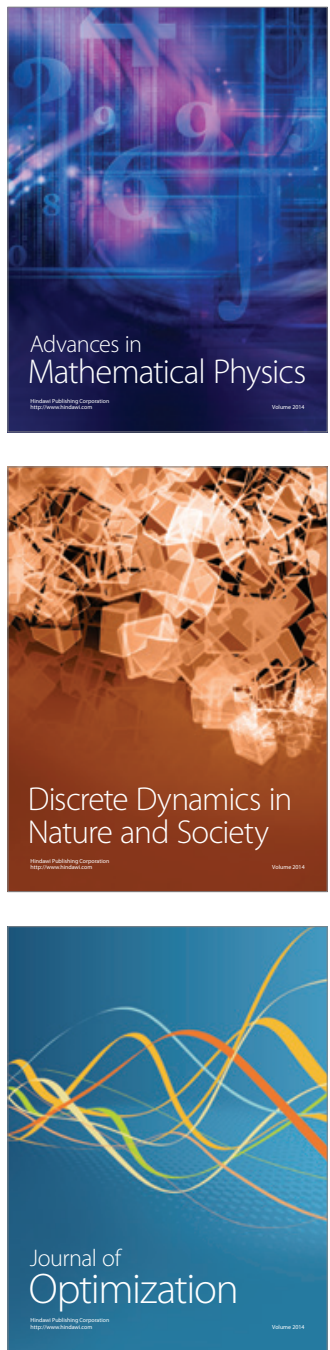